\title{
BMJ Open Relationship between trajectories of post-stroke disability and self-rated health (NeuroAdapt): protocol for a prospective observational study
}

\author{
Sarah K Schäfer (D) , ${ }^{1}$ Robert Fleischmann, ${ }^{2}$ Bettina von Sarnowski, ${ }^{2}$ \\ Dominic Bläsing, ${ }^{1}$ Agnes Flöel, ${ }^{2,3}$ Susanne Wurm ${ }^{4}$
}

To cite: Schäfer SK,

Fleischmann $\mathrm{R}$, von Sarnowski B, et al. Relationship between trajectories of poststroke disability and selfrated health (NeuroAdapt): protocol for a prospective observational study. BMJ Open 2021;11:e049944. doi:10.1136/ bmjopen-2021-049944

- Prepublication history and additional supplemental material for this paper are available online. To view these files, please visit the journal online (http://dx.doi.org/10.1136/ bmjopen-2021-049944).

SKS and RF are joint first authors.

AF and SW are joint last authors.

Received 05 February 2021 Accepted 09 June 2021

Deck for updates

(c) Author(s) (or their employer(s)) 2021. Re-use permitted under CC BY-NC. No commercial re-use. See rights and permissions. Published by BMJ.

For numbered affiliations see end of article.

\section{Correspondence to}

Professor Susanne Wurm; susanne.wurm@med.unigreifswald.de

\section{ABSTRACT}

Introduction Stroke is the leading neurological cause of adult long-term disability in Europe. Even though functional consequences directly related to neurological impairment are well studied, post-stroke trajectories of functional health according to the International Classification of Functioning, Disability and Health are poorly understood. Particularly, no study investigated the relationship between post-stroke trajectories of activities of daily living (ADL) and self-rated health (SRH). However, such knowledge is of major importance to identify patients at risk of unfavourable courses. This prospective observational study aims to investigate trajectories of $A D L$ and SRH, and their modifying factors in the course of the first year after stroke.

Methods and analysis The study will consecutively enrol 300 patients admitted to a tertiary care hospital with acute ischaemic stroke or transient ischaemic attack (TIA; Age, Blood Pressure, Clinical Features, Duration of symptoms, Diabetes score $\geq 3$ ). Patient inclusion is planned from May 2021 to September 2022. All participants will complete an interview assessing ADL, SRH, mental health, views on ageing and resilience-related concepts. Participants will be interviewed face-to-face $1-5$ days post-stroke/ TIA in the hospital; and will be followed up after 6 weeks, 3 months, 6 months and 12 months via telephone. The 12-month follow-up will also include a neurological assessment. Primary endpoints are ADL operationalised by modified Rankin Scale scores and SRH. Secondary outcomes are further measures of ADL, functional health, physical activity, falls and fatigue. Views on ageing, social support, resilience-related concepts, affect, frailty, illness perceptions and loneliness will be examined as modifying factors. Analyses will investigate the bidirectional relationship between $\mathrm{SRH}$ and $\mathrm{ADL}$ using bivariate latent change score models.

Ethics and dissemination The study has been approved by the institutional review board of the University Medicine Greifswald (Ref. BB 237/20). The results will be disseminated through scientific publications, conferences and media. Moreover, study results and potential implications will be discussed with patient representatives.

Trial registration number NCT04704635.
Strengths and limitations of this study

- Prospective study with multiple assessments allowing for the investigation of post-stroke health trajectories.

- Investigation of the complex interplay between activities of daily living and self-rated health in the course of stroke recovery.

- To our knowledge, this is the first study on the predictive value of views on ageing and resiliencerelated concepts for multidimensional post-stroke recovery.

- Limitations include the single-site design and a potential bias due to mostly self-reported data.

\section{INTRODUCTION}

Despite encouraging advances in the early treatment of stroke, ${ }^{1}$ at least one-third of stroke survivors remain with impairments in activities of daily living ( $\mathrm{ADL}^{23}$ ), and thus experience long-term impairment in functional health ${ }^{4}$ and quality of life. ${ }^{5}$ Over $80 \%$ of strokes occur in people aged 65 years and older and outcomes are negatively associated with age. ${ }^{6}$ Stroke causes disability immediately after onset followed by a recovery period up to 3-6 months, after which disability levels tend to stabilise. ${ }^{8}$ However, studies showed that even with similar initial impairment, post-stroke recovery is diverse ${ }^{9}$ : some stroke survivors recover quickly, ${ }^{10}$ others show an accelerated accumulation of disability over time. ${ }^{411}$ More detailed investigations of poststroke health based on the multidimensional concept of the International Classification of Functioning, Disability and Health $\left(\mathrm{ICF}^{12}\right.$; see figure 1) may help identify causes of disproportionate recovery. Previous studies only targeted single dimensions of the ICF concept by investigating trajectories of post-stroke disability, ${ }^{813}$ participation ${ }^{14} 15$ and quality of life. ${ }^{16}$ They identified diverse courses with 


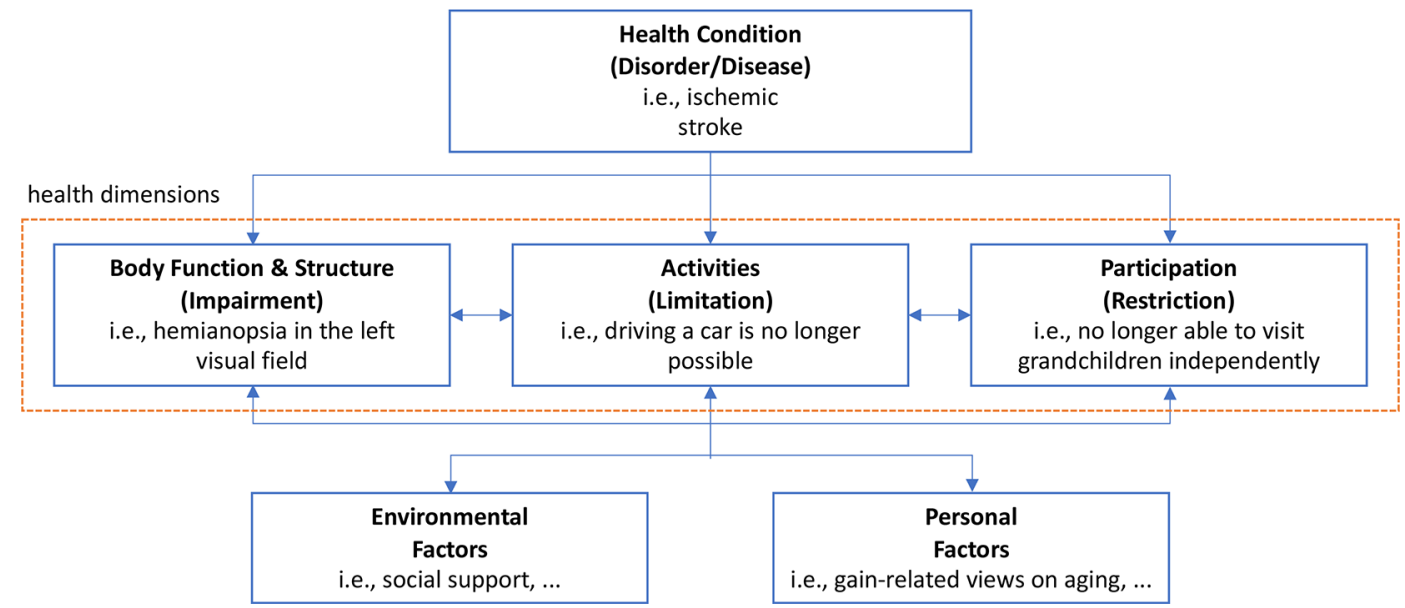

Figure 1 Conceptual framework of the International Classification of Functioning, Health and Disability (World Health Organization, 2001) including an illustrating example for stroke.

respect to speed and directionality ranging from continuous deterioration to substantial improvement. However, none of these studies investigated self-rated health, ${ }^{17}$ which was found to predict post-stroke disability. ${ }^{18}$ Selfrated health can be seen as a subjective global assessment comprising ratings of vitality, physical and mental health, body pain and role functioning. ${ }^{19}$ However, the assessment of self-rated health goes beyond the calculation of a sum score across multiple health domains. Self-rated health is rather a global assessment of various health dimensions that are weighted according to their subjective value for the individual. ${ }^{20}$ This may explain its strong predictive value also for mortality. ${ }^{21}$ Especially, identifying post-stroke self-rated health as an important predictor of ADL over longer periods would be of great clinical importance. Self-rated health as a single-item indicator could be easily used to identify patients at risk of unfavourable courses and could also be an economic tool to assess ongoing recovery processes.

This leads to the question of what can be done to support those who are at increased risk of unfavourable courses. In this context, views on ageing and resiliencerelated concepts (eg, trait-resilience, ${ }^{22}$ sense of coherence $^{23}{ }^{24}$ self-efficacy ${ }^{25}$ ) could be promising starting points. Views on ageing refer to individuals' conceptions about older people, old age and ageing in general (age stereotypes), as well as conceptions of their own ageing and old age (self-perceptions of ageing ${ }^{26}$ ). Resilience describes a positive adaptation process when being faced with trauma, adversity or any other significant thread to one's heath or well-being. ${ }^{27}$ We use the term 'resiliencerelated concepts' as an umbrella term to address trait-like variables that are associated with successful coping; those are trait-resilience, sense of coherence and self-efficacy.

Several studies underline the predictive value of views on ageing for health. ${ }^{26}{ }^{28}$ A recent study shows that negative self-perceptions of ageing and higher subjective age predict incident cardiovascular events. ${ }^{29}$ Moreover, another study found cardiovascular events to negatively impact views on ageing. ${ }^{30}$ Similarly, studies support a strong predictive value of resilience-related concepts for health outcomes. ${ }^{31}{ }^{32}$ One study reported stress resilience in male adolescents to be predictive of stroke, ${ }^{33}$ while another study demonstrated trait-resilience to decline in the first 6 months after ischaemic stroke. ${ }^{34}$ However, to our knowledge, no study yet investigated the predictive value of views on ageing and resilience-related concepts for post-stroke trajectories of self-rated health and ADL. Demonstrating such a predictive value or a modifying influence over time would provide a foundation for prevention and intervention. Both views on ageing ${ }^{35} 36$ and resilience-related concepts ${ }^{37}$ have been shown to be modifiable by brief interventions. Such interventions aiming to strengthen positive view on ageing and resilience could also be promising components of rehabilitation measures after stroke. However, currently there is a lack of evidence base to evaluate such interventions in the context of stroke rehabilitation.

\section{Aim}

Following the multidimensional concept of the ICF, ${ }^{12}$ the study's objective is to investigate trajectories of ADL and self-rated health in the first year after stroke along with their predictors and modifying factors including views on ageing and resilience-related concepts. The study aims at exploring the relationship between self-rated health and ADL, thereby answering the question if self-rated health (and changes in self-rated health) predict ADL (and changes in ADL) and vice versa. Moreover, the study aims to investigate whether trajectories of self-rated health and ADL can be predicted and modified by views on ageing and resilience-related concepts.

\section{METHODS AND ANALYSIS \\ Design}

The study is a prospective observational, hospital-based cohort study in patients with acute ischaemic stroke (AIS) or transient ischaemic attack (TIA) admitted to the Department of Neurology for specialised stroke care 
at the University Medicine Greifswald, Germany. Three hundred patients will be followed for 1 year after AIS /TIA with a total of five assessments. Assessments will take place during patients' hospital stay, after 6 weeks, 3 months, 6 months and 12 months (see table 1 and online supplemental material 1). The 12-month follow-up will also include a neurological assessment at home. Patients will be reimbursed for their efforts. The trial was preregistered at www.clinicaltrials.gov (version 1, 11 January 2021). A Standard Protocol Items: Recommendations for Interventional Trials flow chart ${ }^{39}$ of the study is presented as figure 2 and the completed Strengthening the Reporting of Observational Studies in Epidemiology checklist ${ }^{40}$ can be found as online supplemental material 2.

\section{Department of Neurology at University Medicine Greifswald}

The Department of Neurology is affiliated with a 1000bed tertiary care university hospital. It hence provides all evidence-based imaging and treatment options. This includes multimodal imaging with either CT or MRI. Reperfusion treatment options include intravenous thrombolysis and mechanical thrombectomy. Secondary preventive treatment options provided are stenting and surgical vascular interventions of cerebral arteries, extracranial and intracranial bypass surgery, external ventricular drain and hemicraniectomy. Rehabilitation starts on day 1 with a multidisciplinary team that includes but is not limited to physiotherapy, occupational therapy and speech therapy. Patients are then, depending on their residual impairment following acute treatment, referred to specialised neurological rehabilitation hospitals in the surrounding area. This usually occurs within 1 week after diagnostics are finished. The supraregional stroke unit of the University Medicine Greifswald covers about 500 000 inhabitants within a radius of up to $100 \mathrm{~km}$ including admissions through emergency helicopters.

\section{Patient population: inclusion and exclusion criteria}

Patients with AIS/TIA are consecutively enrolled during their acute treatment (see table 2 for details). In line with exclusion criteria defined for participation in the INSPiRE-TMS Study, ${ }^{41}$ patients with an Age, Blood Pressure, Clinical Features, Duration of symptoms, Diabetes score $<3$ are excluded to reduce the chance of inclusion of patients with stroke mimics. The number of patients with TIA is limited to $20 \%$ to represent common incidence of AIS. ${ }^{42}$

\section{Primary outcome}

Primary outcomes are ADL operationalised by modified Rankin Scale (mRS) scores ${ }^{43}$ and self-rated health (see online supplemental material 1-part A for details on all measures).

\section{Secondary outcomes}

Secondary outcomes are further measures of ADL (ie, Barthel index, ${ }^{44}$ Stroke Impact Scale $16^{45}$ ), physical activity, falls, quality of life, participation, fatigue and mental health.

\section{Modifying factors}

Furthermore, the study investigates factors previously suggested to predict and modify trajectories of ADL and self-rated health. These include views on ageing, social support, resilience-related concepts (ie, trait-resilience, ${ }^{22}$ sense of coherence ${ }^{2324}$ and self-efficacy ${ }^{25}$ ), affect, frailty, illness perceptions and loneliness.

\section{Further medical treatment}

Beginning in the hospital, patients receive various rehabilitation measures tailored to individual impairments. All rehabilitation measures and medical treatments undertaken during the study period will be surveyed and recorded based on medical reports. Therefore, duration and intensity of rehabilitation measures and medical treatments can be taken into account for analyses.

\section{Sample size estimates}

Since no power analysis tool is available for bivariate latent change score modelling, sample size has been estimated based on data simulations using $\mathrm{R}$ (lcsm package). A sample size of 300 including $20 \%$ missing data for ADL and self-rated health was found to be sufficient. Moreover, sample size estimation followed recommendations for latent class mixture models. ${ }^{46}$

\section{Statistical analyses}

Descriptive statistics will be used to describe sample characteristics (ie, age, gender, educational and professional background). Statistical analyses on primary and secondary outcomes will use bivariate latent change score modelling ${ }^{47}$ to investigate the reciprocal relationship between self-rated health and ADL (see online supplemental material A-part B for a schematic illustration). The primary model will use self-rated health and ADL (as assessed by the mRS) as manifest variables measured at all assessment points. Further models will be calculated for self-rated health and physical functioning (ie, Barthel index, ${ }^{44}$ Impact of Stroke Scale ${ }^{45}$ ). Using model comparisons, we will analyse if self-rated health and changes in self-rated health predict changes in ADL, and vice versa. Moreover, we will examine relevant changes in ADL and self-rated health. For both scales, we perceive a one-point shift as clinically meaningful due to rather coarse granularity. ${ }^{48}$ Multiple imputations will be used for missing data completion. The results of the analyses will be presented with and without multiple imputations. Additionally, latent class mixed models ${ }^{49}$ will be used to identify subgroup trajectories for primary and secondary outcomes. We will investigate whether views on ageing and resilience-related concepts at baseline predict different trajectories of selfrated health and ADL and if they play an important role as time-dependent covariate.

\section{Data collection and monitoring}

Outcomes will be collected at all assessment points by trained study team members (ie, doctoral students of medicine and trained study assistants). These trained team members will receive a 2 -week assessment training 
Table 1 Schedule of assessments and measures

\begin{tabular}{|c|c|c|c|c|}
\hline 1-5 days post-stroke & 6 weeks & 3 months & 6 months & 12 months \\
\hline $\begin{array}{l}\text { In-hospital } \\
\text { (T0) }\end{array}$ & (T1) & $\begin{array}{l}80 \\
\text { (T2) }\end{array}$ & (T3) & $\begin{array}{l}\text { o/home } \\
\text { (T4.1/T4.2) }\end{array}$ \\
\hline
\end{tabular}

\section{Sample characteristics}

Demographical variables

Living situation

Vascular risk factors

Living situation
Vascular risk factors

Stroke/TIA severity

$A B C D^{2}$ score

National Institutes of Health Stroke Scale •* (NIHSS)

Cognitive screening (mTICS)

\section{Primary outcomes}

Physical functioning (mRS)

Self-rated health (SF-12)

\section{$\bullet$}

\section{Secondary outcomes}

Physical functioning

\section{Barthel Index}

Stroke Impact Scale 16 (SIS-16)

Physical activity (EHIS-PAQ)

Fear of falling

Quality of life (EQ-5D-5L)

Participation (IMET)

Fatigue (FSS)

Mental health Depression (PHQ-8)

Sleep (PSQI)

Post-traumatic stress (PTSS-14)

\section{Modulators}

Frailty (SHARE-FI)

Loneliness (De Jong Gierveld Loneliness

Scale)

Positive and negative affect (I-PANAS-SF)

Illness perception (B-IPQ)

Views on ageing

Subjective age (DEAS, GEDA/EHIS)

Self-perception of ageing (AgeCog)

Social support (GEDA/EHIS)

Resilience and resilience-related concepts

\begin{tabular}{lllll} 
Trait resilience (RS-13) & $\bullet$ & $\bullet$ & $\bullet$ \\
Sense of coherence (BASOC) & $\bullet$ & $\bullet$ & $\bullet$ \\
Self-efficacy (GSE) & $\bullet$ & $\bullet$ & $\bullet$ & $\bullet$ \\
Scale on healthy lifestyle (SOK) & $\bullet$ & $\bullet$ \\
\hline
\end{tabular}

Continued 


\begin{tabular}{|c|c|c|c|c|}
\hline 1-5 days post-stroke & 6 weeks & 3 months & 6 months & 12 months \\
\hline $\begin{array}{l}\text { In-hospital } \\
\text { (T0) }\end{array}$ & $\begin{array}{l}8 \\
\text { (T1) }\end{array}$ & $\begin{array}{l}\$ \\
\text { (T2) }\end{array}$ & (T3) & $\begin{array}{l}\text { rome/home } \\
\text { (T4.1/T4.2) }\end{array}$ \\
\hline & • & • & • & - \\
\hline
\end{tabular}

Post-traumatic growth (PTGI-10)

${ }^{*}$ Clinical rating.

$A B C D^{2}$ score, Age, Blood Pressure, Clinical Features, Duration of symptoms, Diabetes; AUDIT-C, Alcohol Use Disorders Identification Test-Concise; BASOC, Brief Assessment of Sense of Coherence; B-IPQ, Brief-Illness Perception Questionnaire; DEAS, Deutscher Alterssurvey (German Ageing Survey); EHIS, European Health Interview Survey; EHIS-PAQ, European Health Interview Survey Physical Activity Questionnaire; EQ-5D-5L, European Quality of Life 5 Dimensions 5 Level Version; FSS, Fatigue Severity Scale; GEDA, Gesundheit in Deutschland (German Health Update); GSE, Generalized Self-Efficacy Scale; HSI, Heaviness of Smoking Index; IMET, Index zur Messung von Einschränkungen der Teilhabe (Index for the Assessment of Health-Dependent Participation Restrictions); I-PANAS-SF, International Positive and Negative Affect Schedule Short Form; mRS, modified Rankin Scale; mTICS, modified Telephone Interview for Cognitive Status; NIHSS, National Institutes of Health Stroke Scale; PHQ-8, Patient Health Questionnaire; PSQI, Pittsburgh Sleep Quality Index; PTGI-10, Posttraumatic Growth Inventory 10; PTSS-14, Post-traumatic Stress Scale 14; RS-13, Resilience Scale 13; SF-12, Short-Form Health Survey; SHARE-FI, Frailty Instrument of the Survey of Health, Ageing and Retirement in Europe; SIS-16, Stroke Impact Scale 16; SOK, Selektion, Optimierung, Kompensation-Fragebogen zum gesunden Lebensstil (Selection, Optimization, Compensation Questionnaire on Healthy Lifestyle); TIA, transient ischaemic attack.

(ie, interview training, neurological scoring, etc) and hold an international certificate for the assessment of the mRS and the National Institutes of Health Stroke Scale (NIHSS). Moreover, a random sample of 10 assessments per study team member will be checked by a clinician (ie, neurologist) for rating quality. Data collection will be conducted using the study management system of the Department of Preventive Research and Social Medicine. The system was developed for data collection and management of large-scale survey projects ${ }^{50}$ and offers tools for bedside or home assessments (see table 1, T0 and T4.2) and telephone surveys (see table 1, T1-T4.1). Data will be checked on workdays for protocol conformity and data quality by author DB (see online supplemental material 1-part B for details on data management).

\section{Funding information}

The study receives no external funding.

\section{Patient and public involvement}

The current study aims at investigating differences in multidimensional post-stroke recovery processes according to the ICF. ${ }^{12}$ Self-rated heath is an underresearched yet important outcome of stroke that substantially impacts patients' quality of life, ${ }^{51}$ which will be the focus of this study. Moreover, we are aware that participating in our study can be very exhausting and debilitating for patients, especially in the first days after stroke. Therefore, we will pilot our in-hospital assessment with five patients and adapt the length and design to patients' needs. If the feedback-during piloting or final studynecessitates changes, we will consider these for design and procedure of the study. Assessments regularly include two breaks and will follow a priority list to ensure that data on primary outcomes are complete even when the interview needs to be terminated because it is too strenuous for patients. We also plan to discuss the study results with patient representatives.

\section{Ethics and dissemination}

The study has been approved by the institutional review board of the University Medicine Greifswald (Ref. BB $237 / 20)$. All participants will provide written informed consent in accordance with the Declaration of Helsinki ${ }^{52}$ in its latest revision. In case patients have a legal caregiver, the caregiver will be asked to consent to patients' study participation.

We will use a multimodal approach for dissemination and knowledge transfer. We aim to publish our study results in international peer-reviewed journals with open-access formats to enhance their dissemination. Additionally, study results will be presented at international scientific conferences in the field of Public Health and Neurology. Moreover, we plan to disseminate and communicate our study results using national and-if accessible-international media. Study results and implications will also be discussed with patient representatives aiming at developing tailored interventions for different groups of stroke survivors to promote their quality of life.

\section{TRIAL STATUS}

Due to the COVID-19 pandemic, patient inclusion has been postponed to May 2021 and will approximately end in September 2022. The last patient is expected to be out in September 2023. However, changes to the schedule may be necessary due to the COVID-19 pandemic.

\section{STRENGTHS AND LIMITATIONS}

The current study will provide important insights into trajectories of post-stroke multidimensional health according to the ICF. ${ }^{12}$ To date, studies demonstrated diverse trajectories of disability, ${ }^{8}$ participation ${ }^{14}$ and quality of life ${ }^{16}$ after stroke, but to our knowledge, the current study will be the first to simultaneously analyse individual trajectories of self-rated health and ADL and 


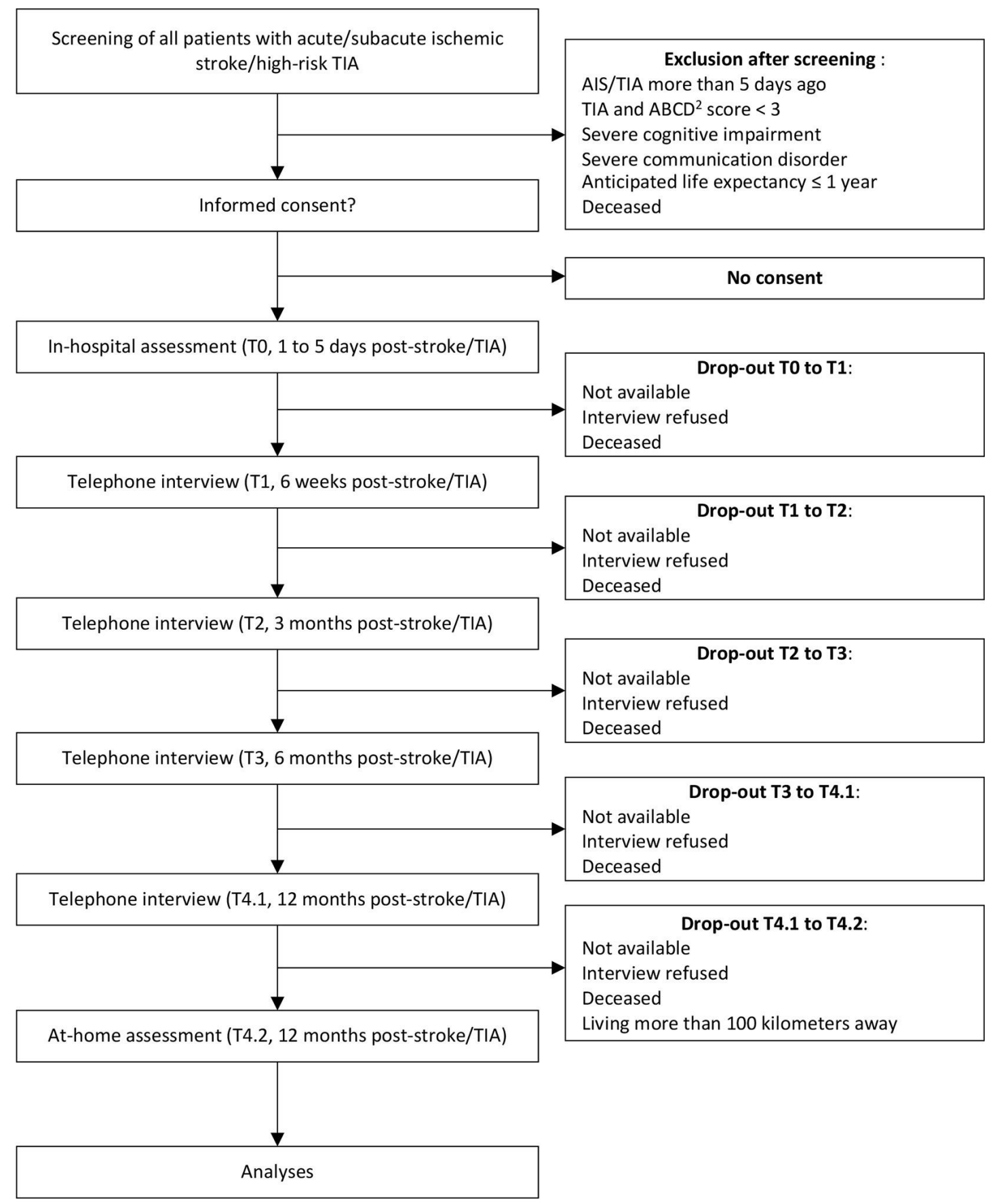

Figure 2 SPIRIT flow chart.

their relationship, as well as possible predictors and modifying factors, in the first year after stroke.

Therefore, the current study will enhance knowledge on dynamics and directions of multidimensional poststroke recovery processes. If self-rated health proves to predict trajectories of ADL, it may help to identify those at risk of unfavourable trajectories of post-stroke multidimensional health and could be useful as an evaluation of ongoing recovery processes. Identifying views on ageing and resilience-related concepts as significant predictors and/or time-dependent modifying variables of trajectories of post-stroke multidimensional health may contribute to the development of tailored secondary prevention measures that could be included in stroke rehabilitation programmes. If our study provides results that can serve as starting points for improving prevention and rehabilitation, future studies should replicate our findings in more diverse patient groups including younger patients and those with cerebral haemorrhage. Developing more tailored secondary prevention and rehabilitation measures will be beneficial both in terms of individual quality of life and on a socioeconomic level to reduce the burden of stroke. ${ }^{3}$

However, it is also important to address the limitations of the current study. First, the study uses a singlesite observational design, so the generalisability of the 


\begin{tabular}{|c|c|}
\hline Inclusion criteria & Exclusion criteria \\
\hline Age $\geq 50$ years & $\begin{array}{l}\text { Severe cognitive } \\
\text { impairment (ie, incomplete } \\
\text { orientation) }\end{array}$ \\
\hline $\begin{array}{l}\text { AIS } / \mathrm{TIA}\left(\mathrm{ABCD}^{2} \text { score } \geq 3\right) \\
\text { Language: fluent in } \\
\text { German }\end{array}$ & $\begin{array}{l}\text { Severe communication } \\
\text { disorder } \\
\text { Index event }>5 \text { days prior } \\
\text { to enrolment } \\
\text { - Anticipated life expectancy } \\
<1 \text { year }\end{array}$ \\
\hline
\end{tabular}

$A B C D^{2}$ score, Age, Blood Pressure, Clinical Features, Duration of symptoms, Diabetes; AIS, acute ischaemic stroke; TIA, transient ischaemic attack.

findings is limited. Future studies will need to replicate the results using multisite designs and larger samples. Second, the study will mostly rely on self-reported data which is both a strength and a limitation of our study. At the one hand, objective data, which will be included in the form of clinical ratings in the in-hospital and home assessments and grip strength measurements (see table 1), are less prone to biases. On the other hand, the study's focus on self-report data and especially selfreported health is considered a strength since subjective perceptions of health and illness were found to be predictive of health outcomes in different populations, ${ }^{5354}$ but are highly under-researched in the context of post-stroke multidimensional health. Moreover, the study uses validated German translations of well-established measures. However, the validation studies did not always include the evaluation of specific psychometric criteria (eg, testretest reliability) for the German version. In these cases, we rely on findings from international studies. Furthermore, our study will use different assessment methods (face-to-face interview at $\mathrm{T} 0$ and telephone interviews at T1-T4.1). This change of assessment method is not ideal, although telephone assessments were found to be valid for mRS assessment. ${ }^{55}$ In case of self-rated health, telephone interviews were found to result in better ratings of current health status compared with assessments via mail. ${ }^{56}$ It is plausible that the change of assessment from face-to-face to telephone interviews may have an impact on patients' ratings in our study, which will be considered when interpreting our study results. However, differences between face-to-face and telephone interviews might be smaller since both assessments represent a personal interview, and potential biases are limited since assessment methods do not differ between patients.

\section{CONCLUSION}

To our knowledge, the current study will be the first to examine the relationship between different dimensions of health in the year after stroke, that is, self-rated health and ADL. Moreover, it will provide evidence whether views on ageing and resilience-related concepts predict and/or modify diverse health trajectories. Therefore, it will improve the identification of risk groups and the development of secondary prevention measures.

Author affiliations

${ }^{1}$ Department of Prevention Research and Social Medicine, University Medicine Greifswald, Greifswald, Germany

${ }^{2}$ Department of Neurology, University Medicine Greifswald, Greifswald, Germany ${ }^{3}$ German Centre for Neurodegenerative Diseases, Greifswald, Germany

${ }^{4}$ Institute for Community Medicine, Department of Social Medicine and Prevention, University of Greifswald Faculty of Medicine, Greifswald, Germany

Acknowledgements We acknowledge support for the Article Processing Charge from the DFG (German Research Foundation, 393148499) and the OpenAccess Publication Fund of the University of Greifswald.

Contributors RF, AF, BvS, SKS and SW (alphabetical order) designed and planned the study. DB designed and implemented the IT infrastructure for the study. SKS drafted the manuscript of the study protocol. RF, AF, BvS and SW gave feedback on the manuscript. All authors approved the manuscript prior to submission.

Funding The authors have not declared a specific grant for this research from any funding agency in the public, commercial or not-for-profit sectors.

Competing interests None declared.

Patient consent for publication Not required.

Provenance and peer review Not commissioned; externally peer reviewed.

Supplemental material This content has been supplied by the author(s). It has not been vetted by BMJ Publishing Group Limited (BMJ) and may not have been peer-reviewed. Any opinions or recommendations discussed are solely those of the author(s) and are not endorsed by BMJ. BMJ disclaims all liability and responsibility arising from any reliance placed on the content. Where the content includes any translated material, BMJ does not warrant the accuracy and reliability of the translations (including but not limited to local regulations, clinical guidelines, terminology, drug names and drug dosages), and is not responsible for any error and/or omissions arising from translation and adaptation or otherwise.

Open access This is an open access article distributed in accordance with the Creative Commons Attribution Non Commercial (CC BY-NC 4.0) license, which permits others to distribute, remix, adapt, build upon this work non-commercially, and license their derivative works on different terms, provided the original work is properly cited, appropriate credit is given, any changes made indicated, and the use is non-commercial. See: http://creativecommons.org/licenses/by-nc/4.0/.

ORCID iD

Sarah K Schäfer http://orcid.org/0000-0001-9885-3252

\section{REFERENCES}

1 Catanese L, Tarsia J, Fisher M. Acute ischemic stroke therapy overview. Circ Res 2017;120:541-58.

2 Feigin VL, Norrving B, Mensah GA. Global burden of stroke. Circ Res 2017;120:439-48.

3 Deuschl G, Beghi E, Fazekas F, et al. The burden of neurological diseases in Europe: an analysis for the global burden of disease study 2017. Lancet Public Health 2020;5:e551-67.

4 Dhamoon MS, Moon YP, Paik MC, et al. Trajectory of functional decline before and after ischemic stroke: the Northern Manhattan study. Stroke 2012;43:2180-4.

5 Dhamoon MS, Moon YP, Paik MC, et al. Quality of life declines after first ischemic stroke. The Northern Manhattan study. Neurology 2010;75:328-34.

6 Chen R-L, Balami JS, Esiri MM, et al. Ischemic stroke in the elderly: an overview of evidence. Nat Rev Neurol 2010;6:256-65.

7 Benjamin EJ, Blaha MJ, Chiuve SE, et al. Heart disease and stroke Statistics-2017 update: a report from the American heart association. Circulation 2017;135:e146-603.

8 Dhamoon MS, Longstreth WT, Bartz TM, et al. Disability trajectories before and after stroke and myocardial infarction: the cardiovascular health study. JAMA Neurol 2017;74:1439-45.

9 Wondergem R, Pisters MF, Wouters EJ, et al. The course of activities in daily living: who is at risk for decline after first ever stroke? Cerebrovasc Dis 2017;43:1-8. 
10 Cassidy JM, Cramer SC, Spontaneous CSC. Spontaneous and Therapeutic-Induced mechanisms of functional recovery after stroke. Trans/ Stroke Res 2017;8:33-46.

11 Edwards JD, Kapral MK, Fang J, et al. Trends in long-term mortality and morbidity in patients with no early complications after stroke and transient ischemic attack. J Stroke Cerebrovasc Dis 2017:26:1641-5.

12 World Health Organization. International classification of functioning, disability, and health. World Health Organization, 2001.

13 Levine DA, Davydow DS, Hough CL, et al. Functional disability and cognitive impairment after hospitalization for myocardial infarction and stroke. Circ Cardiovasc Qual Outcomes 2014;7:863-71.

14 Engel-Yeger B, Tse T, Josman N, et al. Scoping review: the trajectory of recovery of participation outcomes following stroke. Behav Neurol 2018;2018:5472018.

15 de Graaf JA, van Mierlo ML, Post MWM, et al. Long-term restrictions in participation in stroke survivors under and over 70 years of age. Disabil Rehabil 2018:40:637-45.

16 van Mierlo M, van Heugten C, Post MWM, et al. Trajectories of health-related quality of life after stroke: results from a one-year prospective cohort study. Disabil Rehabil 2018;40:997-1006.

17 Spuling SM, Wolff JK, Wurm S. Response shift in self-rated health after serious health events in old age. Soc Sci Med 2017;192:85-93.

18 Araújo Érika de Freitas, Viana RT, Teixeira-Salmela LF, et al. Selfrated health after stroke: a systematic review of the literature. $B M C$ Neurol 2019;19:221.

19 Au N, Johnston DW. Self-assessed health: what does it mean and what does it hide? Soc Sci Med 2014;121:21-8.

20 Arnadottir SA, Gunnarsdottir ED, Stenlund H, et al. Determinants of self-rated health in old age: a population-based, cross-sectional study using the International classification of functioning. BMC Public Health 2011;11:670.

21 DeSalvo KB, Bloser N, Reynolds K, et al. Mortality prediction with a single General self-rated health question. A meta-analysis. $J$ Gen Intern Med 2006;21:267.

22 Connor KM, Davidson JRT. Development of a new resilience scale: the Connor-Davidson resilience scale (CD-RISC). Depress Anxiety 2003;18:76-82.

23 Antonovsky A. Health, Stress, and Coping. Jossey-Bass, 1979.

24 Antonovsky A. Unraveling the mystery of health: How people manage stress and stay well. San Francisco, CA, US: Jossey-Bass, 1987.

25 Bandura A. Self-efficacy: toward a unifying theory of behavioral change. Psychol Rev 1977;84:191-215.

26 Wurm S, Diehl M, Kornadt AE, et al. How do views on aging affect health outcomes in adulthood and late life? explanations for an established connection. Dev Rev 2017;46:27-43.

27 American Psychological Association. The road to resilience, 2014. Available: https://www.apa.org/helpcenter/road-resilience [Accessed 28 Aug 2019].

28 Wurm S, Tesch-Römer C, Tomasik MJ. Longitudinal findings on aging-related cognitions, control beliefs, and health in later life. $J$ Gerontol B Psychol Sci Soc Sci 2007;62:P156-64.

29 Stephan Y, Sutin AR, Wurm S, et al. Subjective aging and incident cardiovascular disease. J Gerontol B Psychol Sci Soc Sci 2021;76:910-9.

30 Wurm S, Wiest M, Wolff JK, et al. Changes in views on aging in later adulthood: the role of cardiovascular events. Eur J Ageing 2020;17:457-67.

$31 \mathrm{Hu} \mathrm{T}$, Zhang D, Wang J. A meta-analysis of the trait resilience and mental health. Pers Individ Dif 2015;76:18-27.

32 Eriksson M, Lindström B. Antonovsky's sense of coherence scale and the relation with health: a systematic review. J Epidemiol Community Health 2006;60:376-81.

33 Bergh C, Udumyan R, Fall K, et al. Stress resilience in male adolescents and subsequent stroke risk: cohort study. J Neurol Neurosurg Psychiatry 2014;85:1331.

34 Zhang W, Liu Z, Zhou X, et al. Resilience among stroke survivors: A cohort study of the first 6 months. J Adv Nurs 2020;76:504-13.

35 Wolff JK, Warner LM, Ziegelmann JP, et al. What do targeting positive views on ageing add to a physical activity intervention in older adults? results from a randomised controlled trial. Psychol Health 2014:29:915-32.

36 Beyer A-K, Wolff JK, Freiberger E, et al. Are self-perceptions of ageing modifiable? examination of an exercise programme with vs. without a self-perceptions of ageing-intervention for older adults. Psychol Health 2019;34:661-76.

37 Joyce S, Shand F, Tighe J, et al. Road to resilience: a systematic review and meta-analysis of resilience training programmes and interventions. BMJ Open 2018;8:e017858.

38 Chmitorz A, Kunzler A, Helmreich I, et al. Intervention studies to foster resilience - A systematic review and proposal for a resilience framework in future intervention studies. Clin Psychol Rev 2018:59:78-100.

39 Chan A-W, Tetzlaff JM, Gøtzsche PC, et al. Spirit 2013 explanation and elaboration: guidance for protocols of clinical trials. $B M J$ 2013;346:e7586

40 von Elm E, Altman DG, Egger M, et al. The strengthening the reporting of observational studies in epidemiology (STROBE) statement: guidelines for reporting observational studies. PLoS Med 2007:4:e296.

41 Ahmadi M, Laumeier I, Ihl T, et al. A support programme for secondary prevention in patients with transient ischaemic attack and minor stroke (INSPiRE-TMS): an open-label, randomised controlled trial. Lancet Neurol 2020;19:49-60.

42 Khanevski AN, Kvistad CE, Novotny V, et al. Incidence and etiologies of stroke mimics after incident stroke or transient ischemic attack. Stroke 2019;50:2937-40.

43 Rankin J. Cerebral vascular accidents in patients over the age of 60. II. prognosis. Scott Med J 1957;2:200-15.

44 Mahoney FI, Barthel DW. Functional evaluation: the Barthel index: a simple index of independence useful in scoring improvement in the rehabilitation of the chronically ill. Maryland State Medical Journal 1965;14:61-5.

45 Duncan PW, Lai SM, Bode RK, et al. Stroke impact Scale-16: a brief assessment of physical function. Neurology 2003;60:291-6.

$46 \mathrm{Kim} \mathrm{S-Y.} \mathrm{Sample} \mathrm{size} \mathrm{requirements} \mathrm{in} \mathrm{single-} \mathrm{and} \mathrm{multiphase} \mathrm{growth}$ mixture models: a Monte Carlo simulation study. Structural Equation Modeling: A Multidisciplinary Journal 2012;19:457-76.

47 Kievit RA, Brandmaier AM, Ziegler G, et al. Developmental cognitive neuroscience using latent change score models: a tutorial and applications. Dev Cogn Neurosci 2018;33:99-117.

48 Kasner SE. Clinical interpretation and use of stroke scales. Lancet Neurol 2006;5:603-12.

49 Magidson J, Vermunt JK. Latent class models. In: The SAGE Handbook of quantitative methodology for the social sciences, 2004: 175-98.

50 Meyer C, Rumpf HJ, Hapke U, et al. Prevalence of alcohol consumption, abuse and dependence in a country with high per capita consumption: findings from the German TACOS study. transitions in alcohol consumption and smoking. Soc Psychiatry Psychiatr Epidemiol 2000;35:539-47.

51 Mavaddat N, Sadler E, Lim L, et al. Perceptions of self-rated health among stroke survivors: a qualitative study in the United Kingdom. BMC Geriatr 2018;18:81.

52 World Medical Association. World Medical association Declaration of Helsinki: ethical principles for medical research involving human subjects. JAMA 2013;310:2191-4.

53 Fong JH, Kok Z-C. Does subjective health matter? Predicting overall and specific ADL disability incidence. Arch Gerontol Geriatr 2020;90:104169.

54 Falconer J, Quesnel-Vallée A. Pathway from poor self-rated health to mortality: explanatory power of disease diagnosis. Soc Sci Med 2017;190:227-36.

55 Janssen PM, Visser NA, Dorhout Mees SM, et al. Comparison of telephone and face-to-face assessment of the modified Rankin scale. Cerebrovasc Dis 2010;29:137-9.

56 Powers JR, Mishra G, Young AF. Differences in mail and telephone responses to self-rated health: use of multiple imputation in correcting for response bias. Aust N Z J Public Health 2005;29:149-54. 